A prot otype aval anche phot odi ode array for scintill ating-fi ber tracking det ect or s

\begin{tabular}{|c|c|}
\hline 著者 & YOSH DA T, SORA T \\
\hline $\begin{array}{l}\text { j our nal or } \\
\text { publ i cat i on titl e }\end{array}$ & $\begin{array}{l}\text { Nucl ear I nst rument s and Met hods in Physi cs } \\
\text { Resear ch Sect i on A }\end{array}$ \\
\hline vol une & 534 \\
\hline nunber & 3 \\
\hline page $r$ ange & $397-402$ \\
\hline year & 200412 \\
\hline URL & ht t p: //hdl . handl e. net /10098/1171 \\
\hline
\end{tabular}




\title{
A prototype avalanche photodiode array for scintillating-fiber tracking detectors
}

\author{
T. Yoshidaa, T. Sora \\ aDepartment of Applied Physics, Faculty of Engineering, University of Fukui, \\ 3-9-1 Bunkyo, Fukui 910-8507, J apan \\ bT he J apan Research Institute, 3-10-19 M inami-Semba, Chuo-ku, O saka 542-0081, \\ J apan
}

17 May 2004

\begin{abstract}
A bstract
We have evaluated the performance of a prototype 16-channel avalanche photodiode (APD) array developed primarily for scintillating-fiber (SCIFI) tracking detectors. The APD array was coupled to a $2.5 \mathrm{~m}$ long SCIFI array, and the detection efficiency was measured for minimum ionizing particles passing through the SCIFI array. The APD array was cooled to $-50^{\circ} \mathrm{C}$ to improve the $S / N$ ratio. We have found that the APD array can read out each individual SCIFI with sufficiently high efficiency.
\end{abstract}

PACS numbers: 29.40.Gx; 29.40.Mc; 42.81.Cn; 85.60.Dw

Keywords: scintillating fiber, avalanche photodiode

(To be published in Nuclear Instruments and M ethods in P hysics Research A) 


\section{Introduction}

Scintillating-fiber (SCIFI) tracking detectors are widely used in high-energy physics experiments these days. The diameter of $0.5-1.0 \mathrm{~mm}$ of a typical SCIFI guarantees good multi-track resolution as well as good spatial resolution. The fast fluorescence decay time of the SCIFI (a few ns) makes fast tracking possible. When a minimum ionizing particle traverses a SCIFI, the number of photons expected at the end of the SCIFI is typically around 20 [1]. Therefore, we need photosensors with high quantum efficiency and high gain to achieve high tracking efficiency. Nowadays, photosensors such as image-intensifier tubes (IITs) [2, 3], position-sensitive photomultiplier tubes (PSPMs) $[4,5]$, and visible light photon counters (VLPCs) [6] are in practical use.

IITs and PSPMs are well-established electron tubes with high electron gain, but their relatively low quantum efficiency $(\approx 25 \%)$ is often insufficient to read out each individual SCIFI efficiently [4]. In this case, one needs to add extra layers of SCIFIs to keep the overall tracking efficiency high. VLPCs are solid state photosensors with high quantum efficiency $(\approx 80 \%)$ as well as high electron gain $(\approx 40000)$. But one often hesitates to use VLPCs, since they have to be operated at ultra-low temperature around $6-7 \mathrm{~K}$ in a cryostat using liquid helium.

We have been testing silicon avalanche photodiodes (APDs), counting on their high quantum efficiency around $80 \%$. Internal electron gain of an APD is usually so small $(\approx 100)$ at room temperature that we cannot expect a sufficient $S / N$ ratio for the small light pulse of a SCIFI. However, it is a well-known fact that the $S / N$ ratio is improved by cooling the APD. Cooling the APD depresses the dark current and reduces the shot noise, since the lower temperature reduces the number of electrons that thermally diffuse from the valence band to the conduction band in the APD. Furthermore, cooling the APD enhances the electron gain of the APD, since the lower temperature reduces the number of phonons that interrupt the electrons in the avalanche process.

In our previous paper [1], we reported our feasibility study made with the Hamamatsu S5343 short-wavelength enhanced type APD. When this APD was operated at room temperature, the detection efficiency for a minimum ionizing particle traversing a SCIFI could not exceed $50 \%$. By cooling it to $-50^{\circ} \mathrm{C}$, however, the detection efficiency reached nearly 100\%. The S5343 APD is a single-channel type. In reality, 
we need APD arrays rather than single-channel APDs in order to realize compact readout of SCIFIs closely lined up in the tracking detectors.

In this paper, we report our subsequent study made with the SPL2368 prototype APD array developed by Hamamatsu Photonics K.K. primarily for compact multichannel readout of SCIFIs. ${ }^{1}$ Fig. 1 shows the SPL2368 APD array. An array consists of $2 \times 8$ APD channels monolithically arranged in $1.62 \mathrm{~mm}$ pitch in a standard dual in-line package. The photosensitive area of each channel is $1 \mathrm{~mm}$ in diameter. The design of this APD was based on the aforementioned S5343 APD. Only the antireflection coating on the APD surface was modified to improve the quantum efficiency for the spectral emission of a 3HF-doped SCIFI ranging from $500 \mathrm{~nm}$ to $600 \mathrm{~nm}$. In this spectral range, the quantum efficiency of the SPL2368 APD array was enhanced to $90 \%$, while that of a standard S5343 APD was $75-80 \%$.

\section{Experimental arrangements}

Fig. 2 shows the schematic diagram of the experimental setup to evaluate the performance of the SPL2368 APD array as a photosensor for SCIFIs. The APD array placed in a small vacuum vessel could be cooled down to $-50^{\circ} \mathrm{C}$ by Peltier devices. Common bias voltage was applied to all the APD channels, since it was not practical to adjust the bias voltage channel by channel. We coupled the APD array to a $2.5 \mathrm{~m}$ long SCIFI array and studied the detection efficiency for minimum ionizing particles penetrating the SCIFI array. The SCIFI array we used is suitable in length for central tracking detectors of today's typical collider experiments.

The structure of the SCIFI array is illustrated in Fig. 3. It consisted of sixteen SCIFIs: Fiber-1 to -16 . The SCIFI we employed was the Kuraray SCSF$3 \mathrm{HF}(1500) \mathrm{M}($ non-S) 3HF-doped multiclad SCIFI $0.75 \mathrm{~mm}$ in outer diameter and $0.66 \mathrm{~mm}$ in core diameter. ${ }^{2}$ First, eight SCIFIs were arranged horizontally in $0.8 \mathrm{~mm}$ pitch. Then, eight more SCIFIs were arranged to fill the gaps between the first eight. The SCIFIs in the array were glued together with white acrylic paint. Each SCIFI in the array was coupled to each APD channel with a $0.2 \mathrm{~mm}$ gap between the polished SCIFI end and the APD surface. No optical interface material was inserted

\footnotetext{
${ }^{1}$ Hamamatsu P hotonics K. K ., 1126-1 Ichino-cho, Hamamatsu City 435-8558, J apan.

${ }^{2} \mathrm{~K}$ uraray Co., Ltd., High Performance Materials Division, 3-1-6, Nihonbashi, Chuo-ku, Tokyo 103-8254, J apan.
} 
in the gap. The holes through which the SCIFIs were drawn into the vacuum vessel were hermetically sealed with epoxy glue. Another end of the SCIFI array was also polished and was terminated by a mirror, the reflectivity of which was $70 \%$.

We used $\beta$-particles as minimum ionizing particles. The SCIFI array was sandwiched between the upper and the lower part of the collimator. Each part of the collimator was made of $8 \mathrm{~mm}$ thick brass. $\mathrm{A}^{90} \mathrm{Sr} /{ }^{90} \mathrm{Y} \beta$-source was placed on the upper part of the collimator. Under the lower part of the collimator, we placed two trigger counters, each of which was a $0.5 \mathrm{~mm}$ thick plastic scintillator. The $\beta$-ray irradiation could be localized almost onto a particular SCIFI through the $0.4 \mathrm{~mm}$ wide slit in the collimator. The length of the slit was $10 \mathrm{~mm}$ along the SCIFI. The collimator could be moved across the SCIFI array by a stepping motor together with the $\beta$-source and the trigger counters. The SCIFI at which the collimator was aimed is referred to as a target SCIFI hereafter.

A coincidence of the two trigger counters produced a trigger signal for data acquisition. A GEANT3-based Monte Carlo simulation shows that the ionizing energy loss of a triggering $\beta$-particle in the SCIFI is equivalent on average to that of a minimum ionizing particle [1].

Signals from the APD array were amplified using a VA32C 32-channel chargesensitive preamplifier chip manufactured by Ideas originally for silicon micro-strip detectors. $^{3}$ The nominal gain of this preamplifier is $60 \mathrm{mV} / \mathrm{fC}$. The nominal peaking time of the preamplifier output signal is $1.5 \mu \mathrm{s}$. The pulse height of the preamplifier output signal was digitized by the Ideas VA-DAQ system.

\section{Experimental results}

Two sample APD arrays (SPL2368 Sample-1 and Sample-2) produced in a batch were evaluated by coupling them to the same SCIFI array one after the other. Each APD array was operated at $-50^{\circ} \mathrm{C}$. The collimator for $\beta$-ray irradiation was set at a distance of $2.3 \mathrm{~m}$ from the APD array along the SCIFIs. The average number of photoelectrons produced in the APD by a triggering $\beta$-particle is expected to be 23 , based on the results of our previous study [1] and the difference in quantum efficiency between the present and the previous APD.

\footnotetext{
${ }^{3}$ Ideas A SA, PO B ox 1, 1330 Fornebu, N orway.
} 
Fig. 4 shows the pulse-height distributions of the preamplifier output signals obtained when Fiber-7 was chosen as a target SCIFI. The distributions for five neighboring SCIFIs including Fiber-7 are presented. The APD array used was SPL2368 Sample-1. The bias voltage applied to the APD channels was 152.0 V. On Fiber-5 and Fiber-9, we see nothing but the noise spectra that are distributed around 0 volt. This implies that almost no $\beta$-particles traversed those SCIFI. Signals produced by $\beta$-particles and obviously larger than the noises are observed on Fiber-6 and Fiber8 , but the percentage of such events is small. On Fiber- 7 that most $\beta$-particles are supposed to have passed through, signals produced by the $\beta$-particles are clearly distinguished from the noise spectrum.

It is because some triggering $\beta$-particles missed the target SCIFI that the noise spectrum appeared on the pulse-height distribution of the target SCIFI such as Fiber-7 in Fig. 4. However, such $\beta$-particles still traversed a SCIFI adjacent to the target SCIFI and produced sizable signals there. We estimate from Fig. 4 that several percent of the triggering $\beta$-particles missed the target SCIFI. Such events also have to be taken into account, when we discuss the detection efficiency. Thus, we define the detection efficiency as a probability that a triggering $\beta$-particle produces a signal higher than a certain threshold in at least one of three adjacent SCIFIs including the target SCIFI in the middle; in case that Fiber-7 is the target SCIFI, for instance, the three adjacent SCIFIs are Fiber-6, Fiber-7, and Fiber-8.

Fig. 5 shows some examples of the bias voltage dependence of the detection efficiency. For each sample APD array, three SCIFIs were randomly chosen as target SCIFIs. Then, for each target SCIFI chosen, the detection efficiency was measured as a function of the bias voltage. The threshold by which signals were distinguished from noises was determined so as to reject $99.5 \%$ of random noises on each APD channel at each bias voltage. Each efficiency curve in Fig. 5 steadily goes up to nearly $100 \%$ as the bias voltage increases, and forms a plateau lasting for about 3 volts. When the bias voltage exceeds $152.0 \mathrm{~V}$, however, the detection efficiency begins to decrease, since the APD begins to break down and the threshold rises more rapidly than the signals.

Fig. 6 shows the uniformity in detection efficiency across the SCIFI array. The APD arrays were operated at a bias voltage of $151.5 \mathrm{~V}$. The collimator was moved by the stepping motor to irradiate all the SCIFIs one by one, and the detection efficiency 
was measured as a function of the collimator position. In this measurement, we raised the threshold so as to reject $99.9 \%$ of random noises on each APD channel. In addition to Fig. 6, the average pulse height of the preamplifier output signals from each target SCIFI is presented in Fig. 7, where we used events in which a signal higher than the threshold was generated only by the target SCIFI among three adjacent SCIFIs including the target SCIFI in the middle. This event selection guaranteed that the triggering $\beta$-particle passed near the center of the target SCIFI. The 32 APD channels tested here vary in average pulse height from $160 \mathrm{mV}$ to $700 \mathrm{mV}$. In spite of such a large variation in pulse height, the detection efficiency is high enough $(\approx 97 \%)$ everywhere except near the edges of the SCIFI array where some of the triggering $\beta$-particles hit no SCIFIs.

\section{Summary}

We have experimentally demonstrated that the SPL2368 prototype APD array is a promising photosensor for SCIFI tracking detectors. Performance of the APD array as a photosensor for SCIFIs was evaluated using a $2.5 \mathrm{~m}$ long SCIFI array built with 3HF-doped multiclad SCIFIs $0.66 \mathrm{~mm}$ in core diameter. The sample APD arrays, when operated at $-50^{\circ} \mathrm{C}$, could read out each individual SCIFI with sufficiently high efficiency for minimum ionizing particles. Our next efforts will be directed towards applying the APD arrays to SCIFI tracking detectors on a large scale.

\section{A cknowledgements}

We thank Y. Ishikawa and T. Inuzuka from Hamamatsu Photonics K.K. for their useful advice concerning the APD array. We also thank T. Okusawa and K. Yamamoto from Osaka City University for many useful discussions and suggestions. This work was supported in part by a Grant-in-Aid for Scientific Research on Priority Areas from the Ministry of Education, Science, Sports and Culture of Japan. 


\section{R eferences}

[1] T. Okusawa, Y. Sasayama, M. Yamasaki, T. Yoshida, Nucl. Instr. and Meth. A 459 (2001) 440 .

[2] E. Eskut et al., Nucl. Instr. and Meth. A 401 (1997) 7.

[3] B. J. Kim et al., Nucl. Instr. and Meth. A 497 (2003) 450.

[4] I. Daito et al., Nucl. Instr. and Meth. A 433 (1999) 587.

[5] B. Adeva et al., Nucl. Instr. and Meth. A 515 (2003) 467.

[6] A. Bross et al., Nucl. Instr. and Meth. A 477 (2002) 172. 


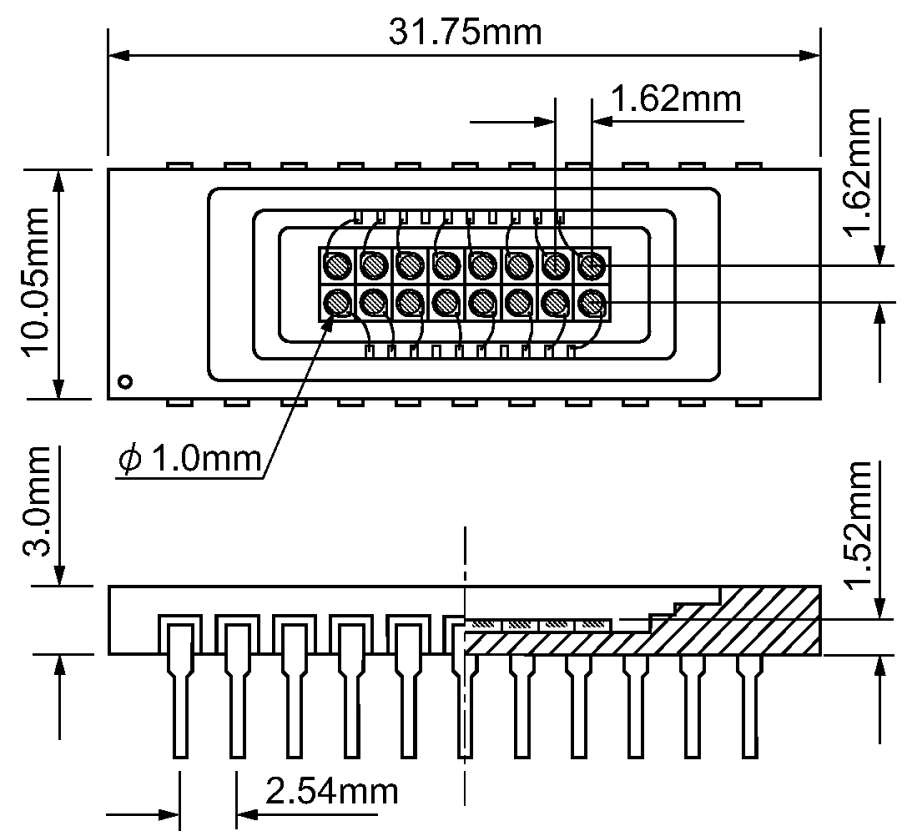

Fig. 1. The SPL2368 prototype APD array. Shaded circles are the photosensitive surfaces.

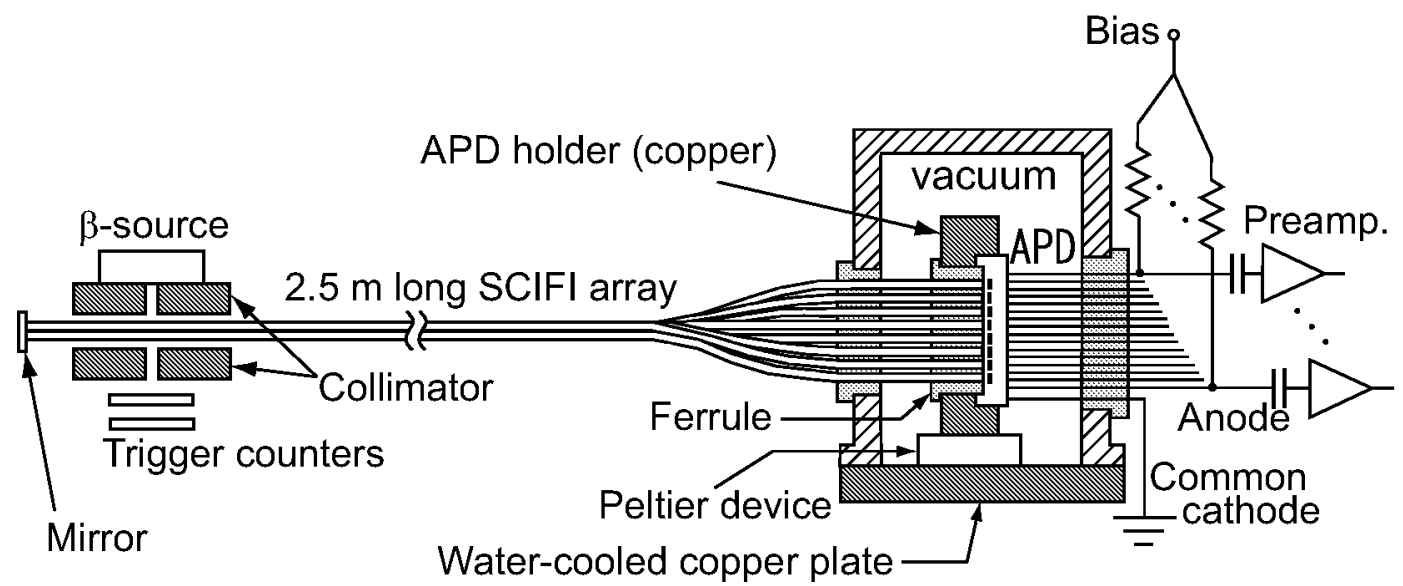

Fig. 2. The schematic diagram of the experimental setup. 


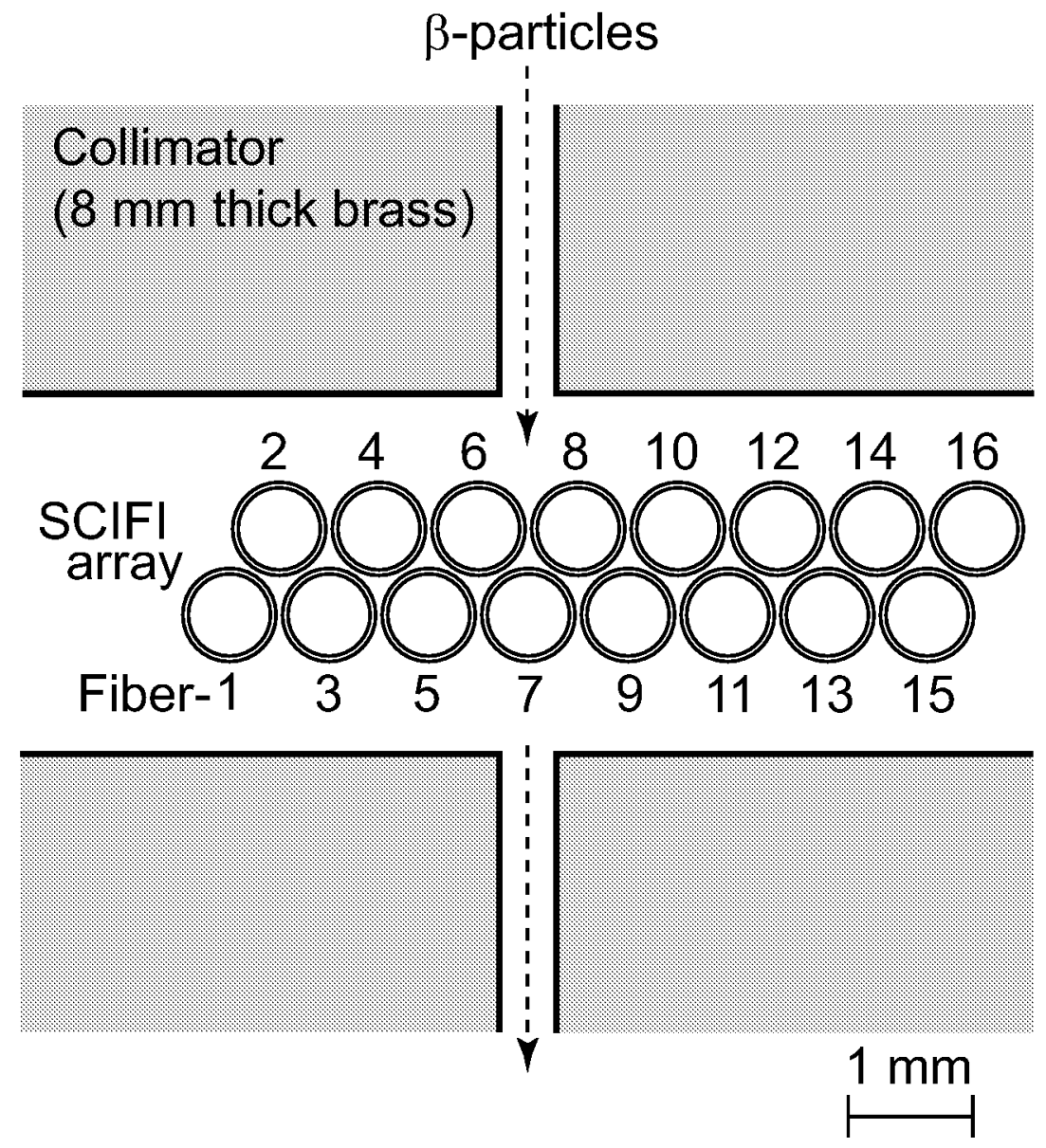

Fig. 3. A cross-section of the SCIFI array and the collimator. 


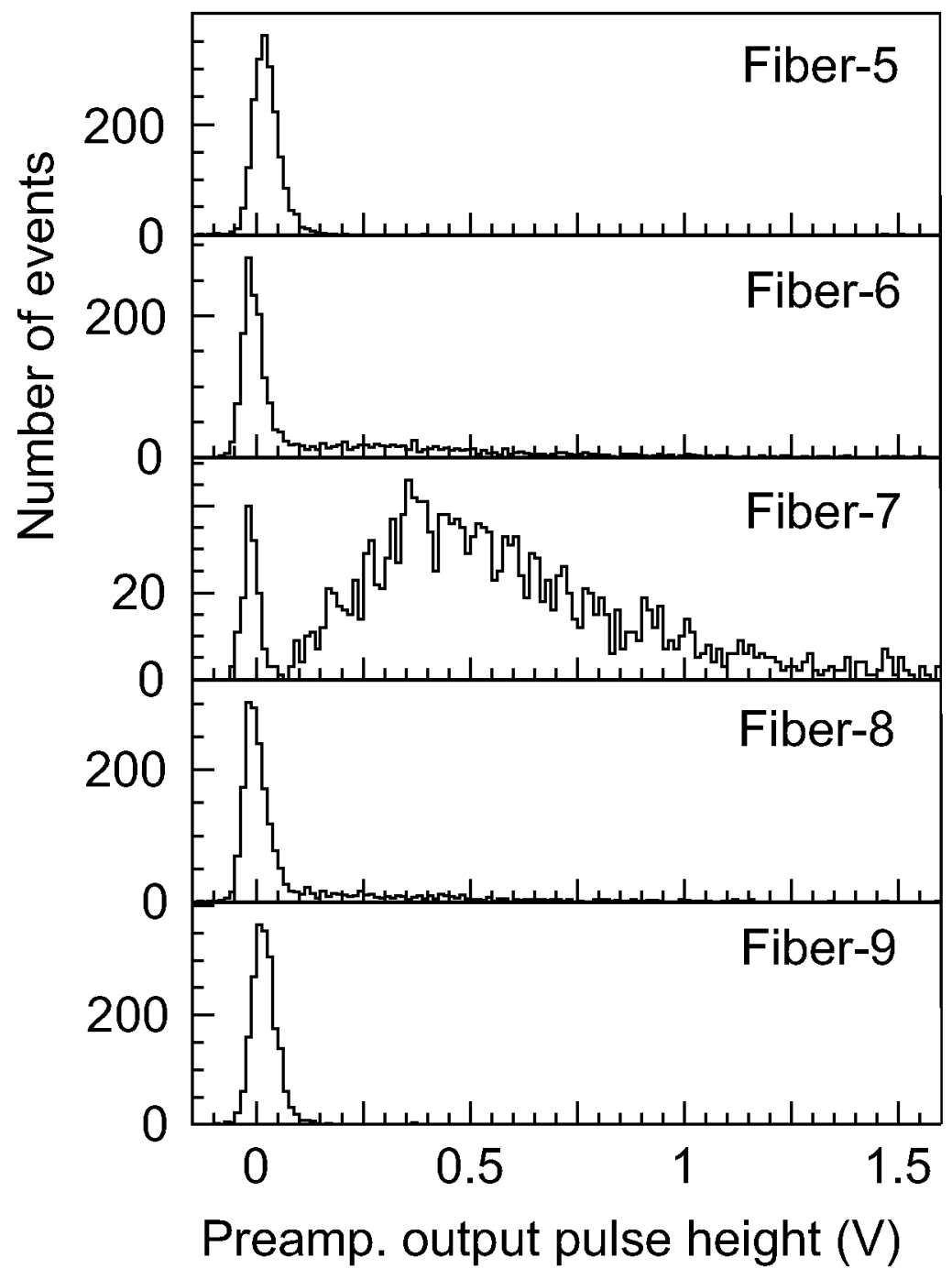

Fig. 4. The pulse-height distributions of the preamplifier output signals for five neighboring SCIFIs. The collimator was aimed at Fiber-7. The APD array used was SPL2368 Sample-1. 


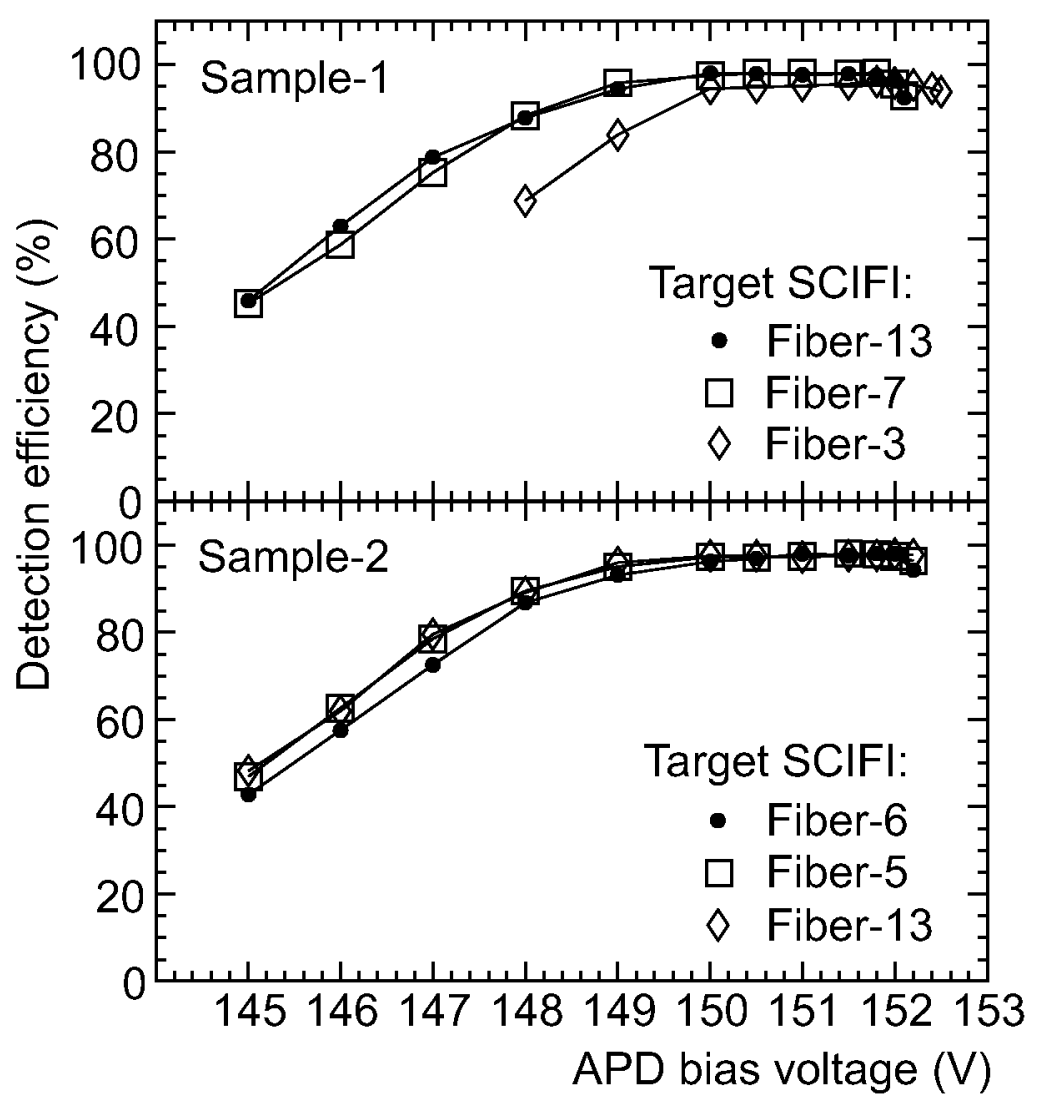

Fig. 5. Bias voltage dependence of the detection efficiency. Target SCIFIs were randomly chosen for each of the sample APD arrays (SPL2368 Sample-1 and Sample2). 


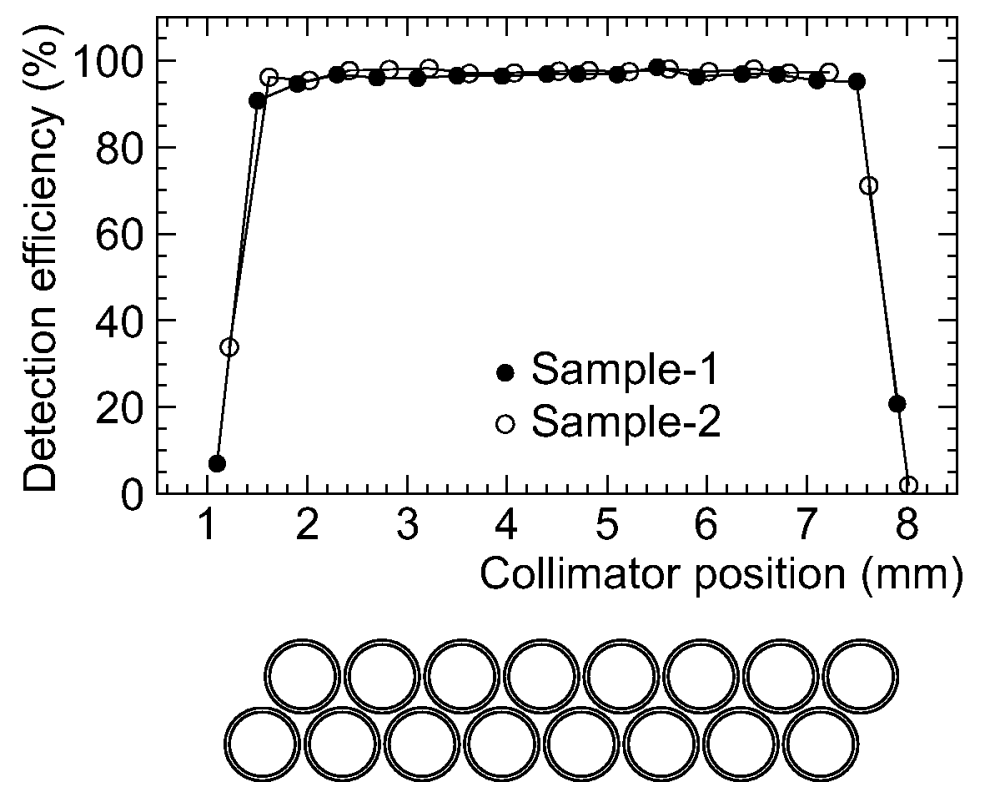

Fig. 6. Detection efficiency as a function of the collimator position for two sample APD arrays (SPL2368 Sample-1 and Sample-2). The location of each SCIFI is also indicated below the graph.

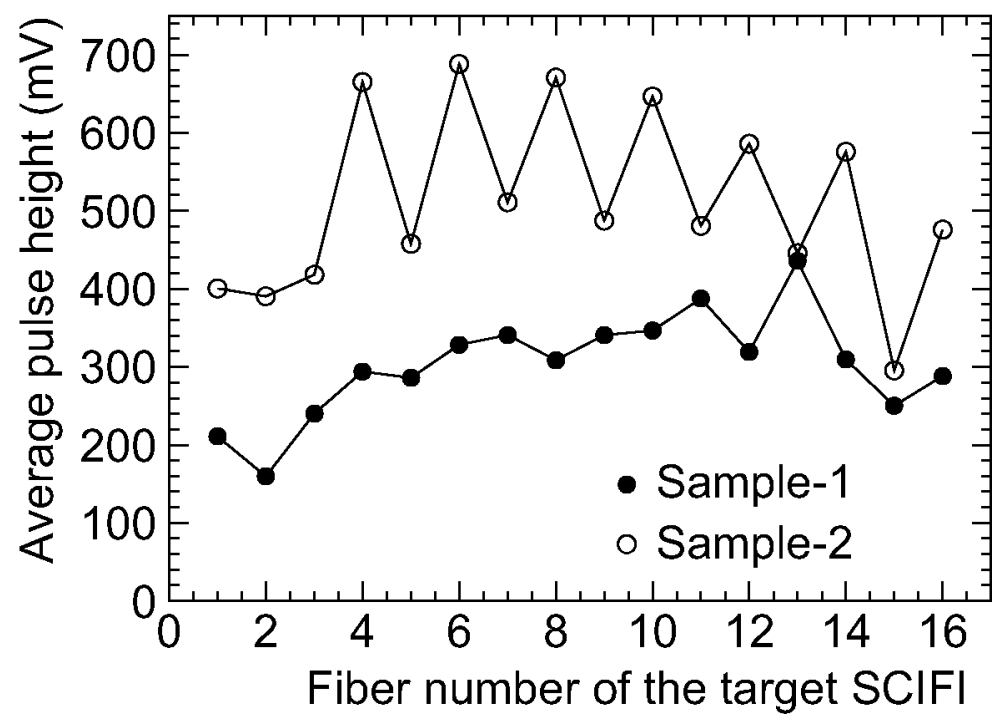

Fig. 7. Average pulse height for each target SCIFI measured with two sample APD arrays (SPL2368 Sample-1 and Sample-2). 